\title{
Analog Pulse Shape Discrimination Based on Time Duration and Pulse Height
}

\author{
R. Harn ${ }^{1}$, A. Osovizky ${ }^{1,2}$, Y. Kadmon ${ }^{1}$, A. Manor ${ }^{1}$, and M. Ghelman ${ }^{1}$ \\ ${ }^{1}$ Nuclear Research Center - Negev, Israel \\ ${ }^{2}$ Radiation Detection Department, Rotem Industries Ltd, Israel \\ RonH@nrcn.org.il
}

\begin{abstract}
Pulse shape discrimination is a name of a group of techniques used to detect and distinguish between different types of radiation interactions. Analog pulse shape discrimination methods can be more suitable than digital methods, for high-speed scintillators both from rate and power consumption perspectives. Common analog discrimination methods are based on pulse-height and pulseenergy discrimination techniques. Other techniques rely on the time difference in the pulse width such as the ZeroCrossing methods. Neither of the above combine both amplitude and time methods. We present a novel analog pulse shape discrimination topology that combines both height and time domain. The topology is based on discrimination according to the pulse duration in time combined with compensation function of the pulse height. Amplitude of the pulse is used as a restraining factor. Subsequently, our topology correctly identifies fast pulses that are prolonged in time due to their high amplitude. The topology yields superior discrimination capabilities, under degraded light collection conditions, with an uncertainty gap smaller than $1 \mathrm{~ns}$ in pulse width. The ability to control both the time and the amplitude parameters individually, provides tailored adjustment for various detectors and pulse shape discrimination applications.
\end{abstract}

Keywords - PSD, Discrimination, Classification, PHA, Plastic Scintillator.

\section{INTRODUCTION}

$\mathrm{P}$ ULSE shape discrimination (PSD) is a general name for a group of techniques, aimed to discriminate between different types of radiations, generally used to distinguish between neutrons and gamma radiation, and between alpha and beta radiation. The core of all PSD techniques is the identification of differences in the nuclear pulse shape generated by the detector for different radiations. In order to increase variation in the generated pulse shape, when using scintillator-based detectors, an integration of scintillators is often used. For example, $\mathrm{ZnS}(\mathrm{Ag})$ and plastic scintillators are often combined for alpha and beta radiation discrimination. Moreover, different scintillator structures can be used to enhance the pulse shape deviation, such as in the phoswich detector [1].
Different PSD methods vary in their discrimination capabilities, detection efficiency, and power consumption. PSD methods can be categorized into two groups, analog-based techniques, and digital techniques. In the last two decades, the development of high-speed acquisition hardware such as high frequency analog to digital converters (ADC) and field programmable gate arrays (FPGA) gave the digital approach significant advantages over traditional analogue PSD techniques [2]. Digital PSD methods enable simultaneous analysis of a large number of different parameters. Furthermore, digital PSD methods enable implementation of complex algorithms, providing superior discrimination capabilities, as compared to analog techniques [3]. Digital PSD discrimination capabilities improved even further with the development of the artificial intelligence field [4,5]. However, complex algorithms require long processing times, thereby limiting the count rate and increasing the dead time. Sampling of nuclear signals, especially from scintillators with fast decay constants, requires fast samplers, which have significant power consumption. For this reason, an analog PSD topology can be more suitable when working with fast decay scintillators, especially for battery-based applications. The analog methods can be divided into two categories: classification by height (pulse height discrimination - PHD), and classification by time (pulse width discrimination - PWD). PHD is based on classification either by the pulse height or by pulse charge [6]. Classification by time is based on discrimination by the duration of the pulse, using the pulse width over a defined threshold level [7]. Under degraded light collection conditions, such as long and opaque scintillators, a lower pulse-height is obtained, while the noise level is unaffected. Consequently, lower signal to noise ratio is obtained, causing amplitude-based methods to yield a considerable number of misclassifications. Contrary to pulse height and energy that decrease proportionally to the light collection efficiency, the pulse shape is less affected. However, time and pulse width-based methods are not error-proof either. Pulse width at over threshold of fast and high amplitude pulses can have a similar width as slower pulses with lower amplitude.

In this work a novel analog PSD topology that overcomes this issue is presented. The topology is based on discrimination according to the pulse duration in time, combined with a compensation as a function of the pulse height. The amplitude 
of the pulse is used as a restraining factor. Subsequently, our topology correctly identifies fast pulses that are prolonged in time due to their high amplitude. The topology was realized using high speed printed circuit board and was found to present superior discrimination capabilities with an uncertainty gap smaller than $1 \mathrm{~ns}$ in pulse width. The ability to control both time and amplitude parameters separately provides adjustment for various detectors and PSD applications. The topology is introduced in two stages. In sections II and III, a time durationbased PSD is introduced, in section IV and V the combined time and pulse height PSD topology is presented.

\section{ANALOG PSD BASED ON TIME DURATION}

As mentioned, when the detector's light collection efficiency is reduced, for example when radiation-scintillator interaction occurs in the corner of the detector, the probability of a misclassification increases. This is due to the strong correlation between the light collection efficiency and the pulse energy. Alpha and beta pulses obtained from a plastic and $\mathrm{ZnS}(\mathrm{Ag})$ scintillator coupled to a photomultiplier tube (PMT) are shown in Fig. 1 (a) and (b) respectively. While in the amplitude domain there is an overlap between the alpha and the beta pulse height between $100 \mathrm{mV}$ to $150 \mathrm{mV}$, their time decay constants intervals are separated.

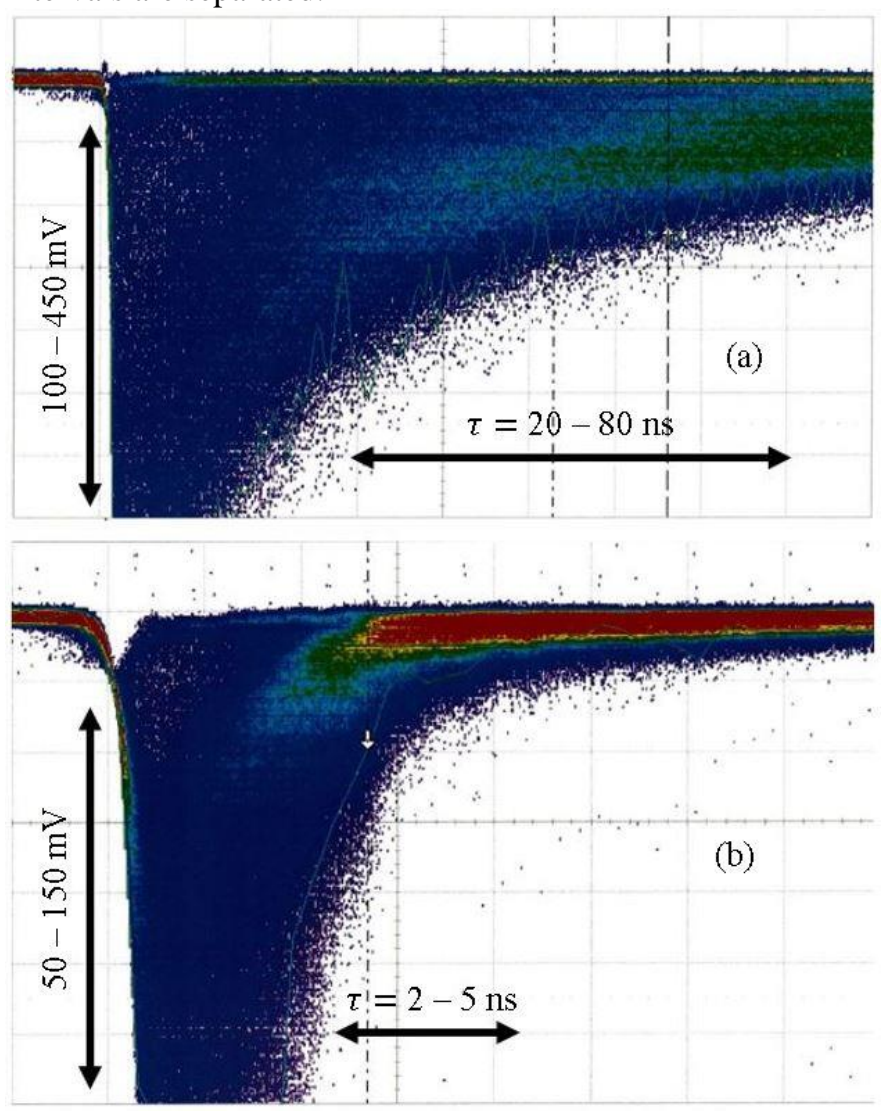

Fig. 1. Alpha pulses (a) and beta pulses (b) obtained from a detector based on plastic and $\mathrm{ZnS}(\mathrm{Ag})$.

Hence, for a radiation detector that produces the pulses shown in Fig. 1, separation based on time domain will yield less misclassifications than pulse height discrimination techniques. The distribution of the pulses on the amplitude - decay time span is shown in Fig. 2. As seen, a vertical separation by the time will yield a better separation compared to a horizontal separation, based on amplitude discrimination.

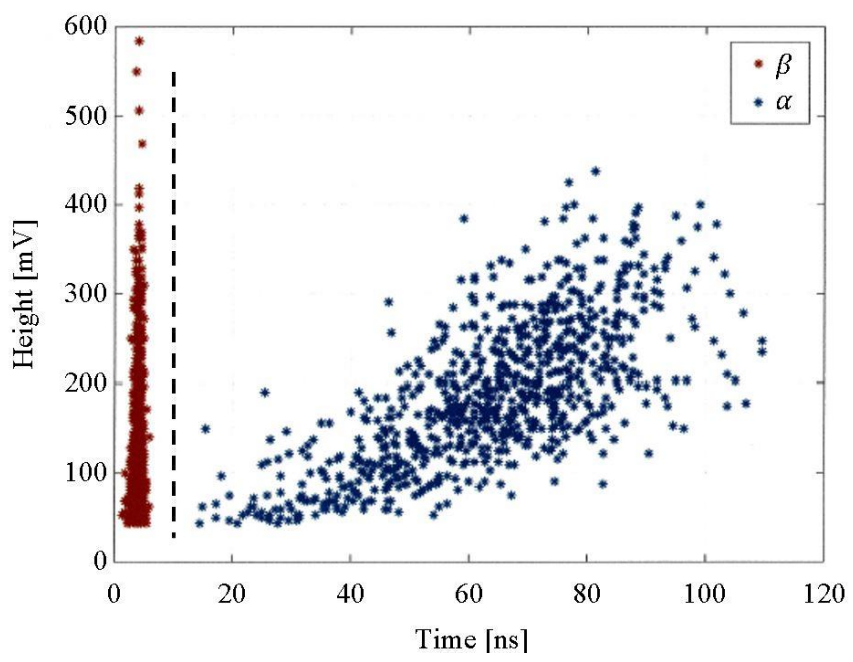

Fig. 2. The distribution of the alpha and beta pulses over the amplitude-decay time span.

A scheme of the proposed time-based discrimination topology is shown in Fig. 3. The nuclear pulse is fed from the detector to a high bandwidth amplifier. The pre-amplifier's bandwidth should be sufficient in order to preserve the detector's pulse shape. An attenuation of the high frequency components at the nuclear signal will increase the resemblance between the fast and slow pulses, hence, increasing misclassification probability. In addition, the amplifier should have high amplification and saturation recovery capabilities, in order to obtain a full width of the pulse over the threshold detection level. Higher pulses, beyond threshold, generate a more linear connection between the width of the amplified pulse, $T_{W}$, and the pulse decay constant, $\tau_{f}$. On the other hand, wide bandwidth amplifies generate higher noise levels, and hence necessitate higher threshold detection levels. Therefore, the input operational amplifier should be carefully selected, by referring to its gain-bandwidth product and its noise level, compared to the detector's pulse shape and internal noise. The amplifier output is fed into a comparator level that generates a digital pulse. The digital pulse width, $T_{W}$, matches the amplifier output pulse width. Hence, the time domain property of the detector pulse is separated. The comparator functions as a low-level discriminator (LLD). Therefore, all the pulses that exceed the threshold detection level is counted at the "ALL" output port, meaning it is either a slow pulse (neutron / alpha), or a fast pulse (beta / gamma). Therefore, the topology detection efficiency depends on the detection threshold level. Hence, the threshold is set as low as possible as a function of the detector noise level. The comparator output controls a single pole double through (SPDT) analog switch. The SPDT switch inputs are connected to the circuit analog power supply, VCC, and to the circuit ground potential. The SPDT switch output is fed into an integrator. Therefore, when comparator output is high the integrator is fed with a regulated stable voltage, VCC, instead of the high output of the comparator that deviate from one 


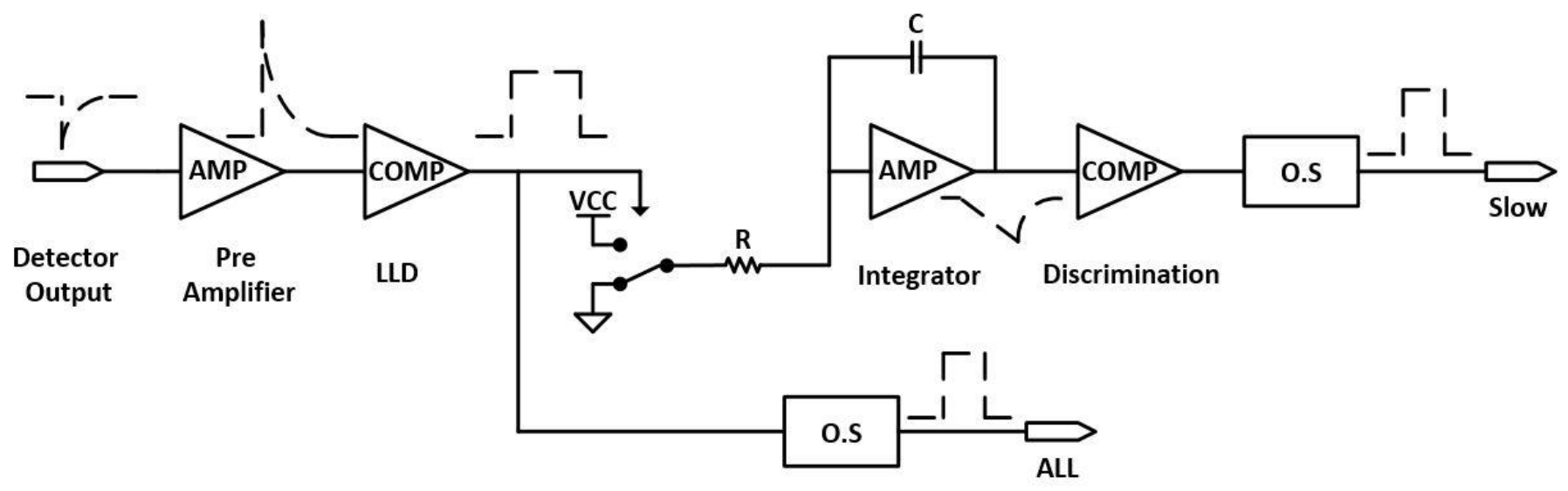

Fig. 3. Scheme of the proposed time based discrimination topology.

component to the other. When the comparator output is low the integrator is fed with the ground potential instead of the comparator's low-level voltage. The output of the integrator peak has a linear dependence in the digital pulse width, $T_{W}$, as shown in (1),

$$
V_{\text {Integrator }}=-\frac{V C C}{R C}\left(T_{W}+\tau_{\text {Const }}\right)
$$

Where, $\mathrm{R}$ and $\mathrm{C}$ are the integrator resistor and capacitor respectively. The constant $\tau_{\text {Const }}$ is a component added due to the switch parasitic junction capacitance discharge decay time. The integrator output is fed into a discrimination comparator with an adjustable threshold. By adjusting the discrimination comparator threshold, the width that classifies between fast and slow pulses is determined. The signal shapes at different nodes of the electronic topology are shown in Fig. 4 (a), for a slow pulse with a decay time constant of $20 \mathrm{~ns}$, and for a fast pulse, Fig. 4 (b), with a decay time constant of $10 \mathrm{~ns}$.
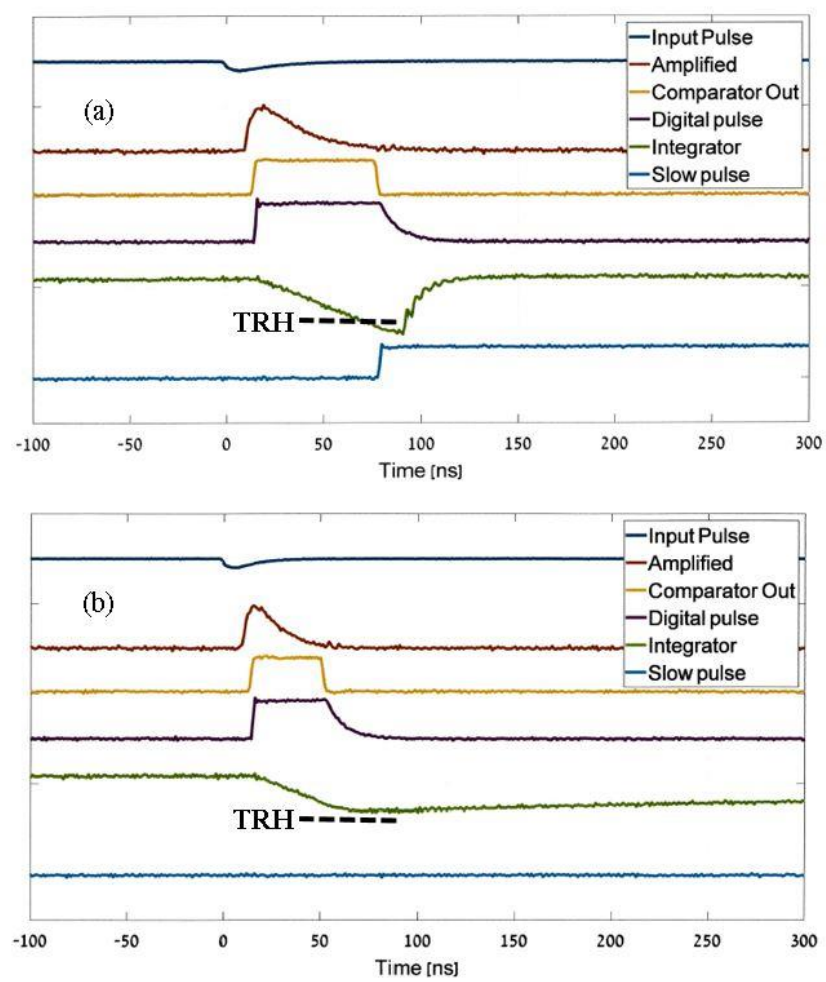

Fig. 4. Signal shape at different nodes, (a) for a slow pulse, (b) for a fast pulse.
Once the integrator curve exceeds the threshold, the signal is detected as a slow pulse, and the "Slow" signal rises. A discharge logic is enabled to implement a fast discharge of the integrator capacitor, in order to enable the process of a new pulse, hence reducing the dead time.

\section{TIME DURATION PSD RESUltS}

The topology was implemented on a FR-4 PCB designed for high frequency operation. The topology was evaluated using CAENs DT5810 fast digital emulator. The time width threshold was adjusted to $50 \mathrm{~ns}$. The topology input was fed with pulses with different decay constants at four amplitudes, 100, 200, 400 , and $800 \mathrm{mV}$. At each point, the percentage of slow classifications were recorded. The result is presented in Fig. 5.

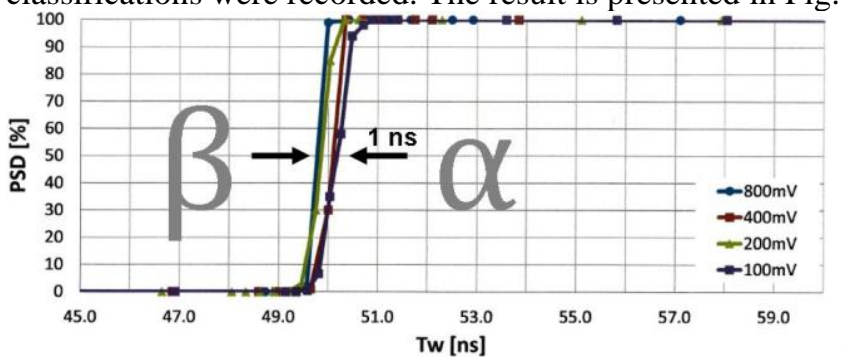

Fig. 5. Percentage of "Slow" classification for different pulses widths for four amplitudes, classification width threshold is set to $50 \mathrm{~ns}$.

As seen in Fig. 5, pulses wider than $50.5 \mathrm{~ns}$ were all classified as slow pulses, while all pulses narrower than $49.5 \mathrm{~ns}$ were classified as fast pulses, in accordance with the $50 \mathrm{~ns}$ threshold. The 1 ns gap where classification is not certain is the uncertainty gap. One can note that the higher the input amplitude, the smaller the uncertainty gap. This is because of the improvement in the linear connection between the pulse width and the pulse decay time with amplitude growth. Furthermore, the congestion of the curves regardless of their amplitude shows that there is a very small amplitude dependence, as desired in a time-based amplitude PSD. Experimental results for the vertical separation line presented in Fig. 2. is shown in Fig 6. The reduction in the linear pulse width - decay constant connection can be observed for small amplitudes. 


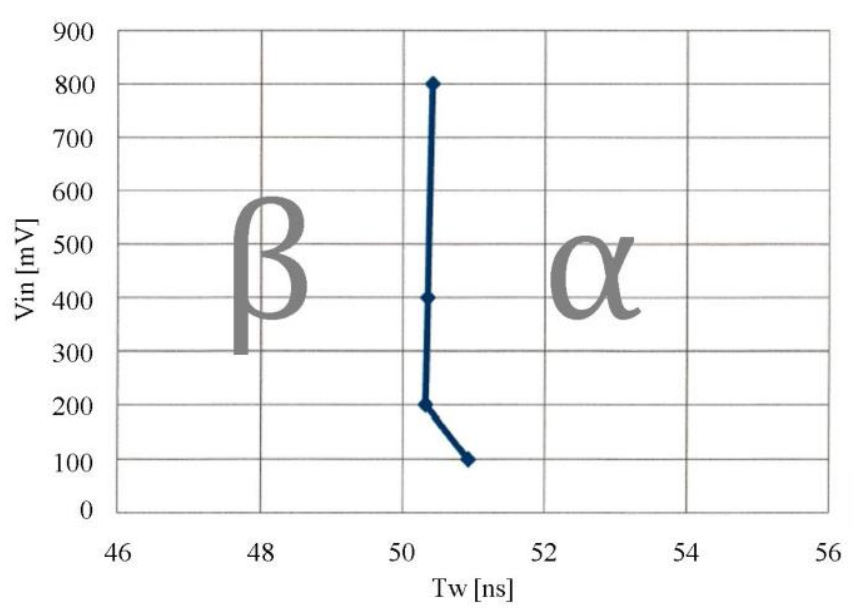

Fig. 6. Measured results for the vertical classification line in the height - time span produced by the time duration PSD topology.

\section{AnAlog PSD BASED ON TIME DURATION AND PULSE HEIGHT COMBINATION}

As mentioned, time duration classification techniques are not error proof. Light collection efficiency reduction can cause misclassifications between fast pulses that are prolonged in time due to their high amplitude, and between slow attenuated pulses. A detector based on a $30 \mathrm{~mm}$ X $30 \mathrm{~mm}$ X $1 \mathrm{~mm}$ plastic scintillator combined with a $\mathrm{ZnS}(\mathrm{Ag})$ plating was assembled.

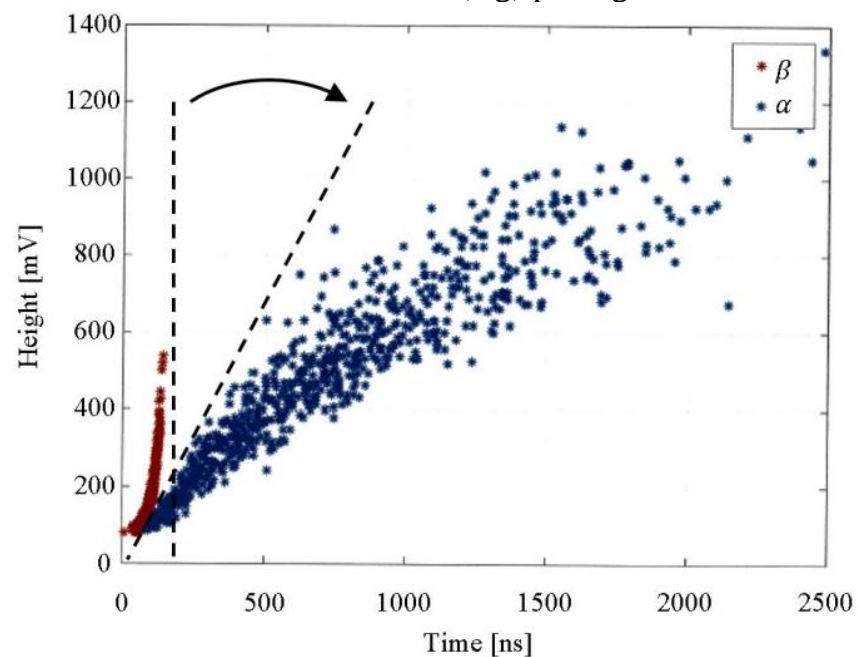

Fig. 7. The distribution of the alpha and beta pulses over the amplitude-decay time span in a single SiPM detector.
The scintillator was coupled to a single $6 \mathrm{~mm}$ X $6 \mathrm{~mm}$ silicon photodiode (SiPM) with a $10 \mathrm{~mm}$ light guide. Pulse distribution on the amplitude time with span is presented.

It can be noticed that in the case presented in Fig. 7, both PHD and PWD techniques will generate a significant number of misclassifications. However, a diagonal classification line, as shown in Fig. 7, can reduce the number of misclassifications. In order to produce such a diagonal separation line, a forward feedthrough was added to the topology from section II, as shown in Fig. 8. The time and amplitude combined topology implement a forward feed of the inverse pulse via a feedforward resistor, $\mathrm{R}_{\mathrm{F}}$. Hence, higher pulses will generate stronger discharge current that discharge the integrator capacitor simultaneously to its charging process. Hence, higher pulses would have to be prolonged in time, compared to lower pulses, in order to be classified as a slow pulse. The angle of the diagonal separation line is a function of the discharge current with respect to the integrator charging current. Hence, the angle is a function of the ratio $R_{F} / R$.

\section{RESULTS}

The discrimination curves of the height-time combination topology for 100, 200, 400, and $800 \mathrm{mV}$ is presented in Fig. 9. Compared to the time-based discrimination presented in Fig. 5, the curves are not grouped together as expected, as the discrimination depends on the amplitude of the pulse. As seen from Fig. 9, for higher pulse amplitudes, larger values of $T_{W}$ are required in order for the classification to reach the slow region. Furthermore, as opposed to the time-duration topology, where higher amplitude generated smaller uncertainty gaps, in the height-time combination topology the opposite is obtained. This is because in the combined topology the input is fed directly to the integrator input. Hence, injected noise is integrated. Higher pulses require longer integration time, hence more noise is integrated, increasing the uncertainty gap. Measured results for the diagonal classification line yield using the time domain and pulse height combination are shown in Fig. 10. The blue curve is the diagonal classification line obtained from the combine amplitude-time topology while the red curve is the classification line based only on time domain. As can be seen, the results match the desired separation diagonal curve shown in Fig. 7.
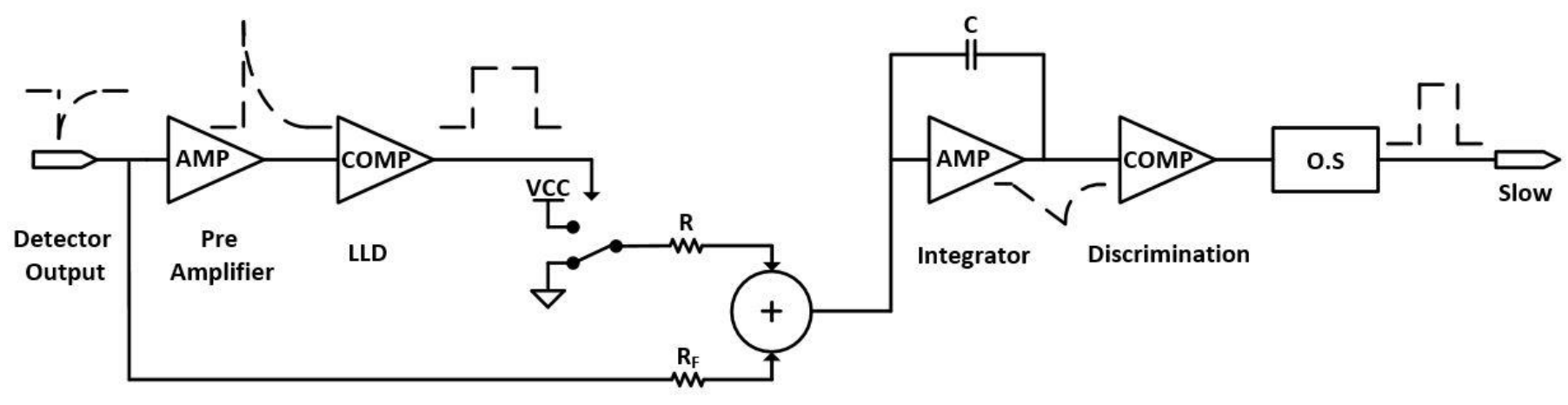

Fig. 8. Scheme of the proposed time duration and pulse height discrimination topology. 


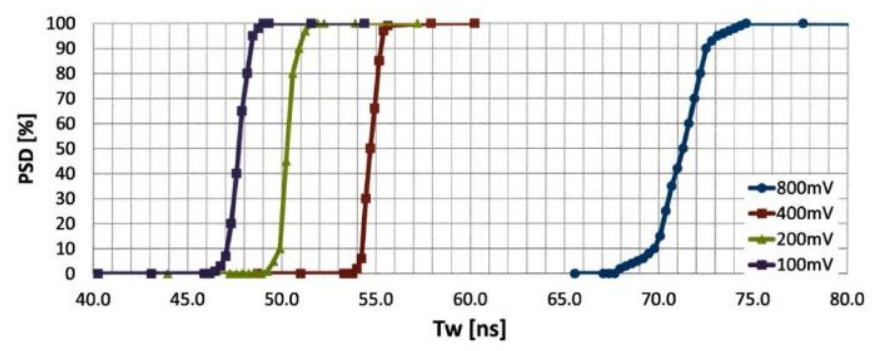

Fig. 9. Percentage of "slow" classification for different pulses widths using time duration and pulse height discrimination topology.

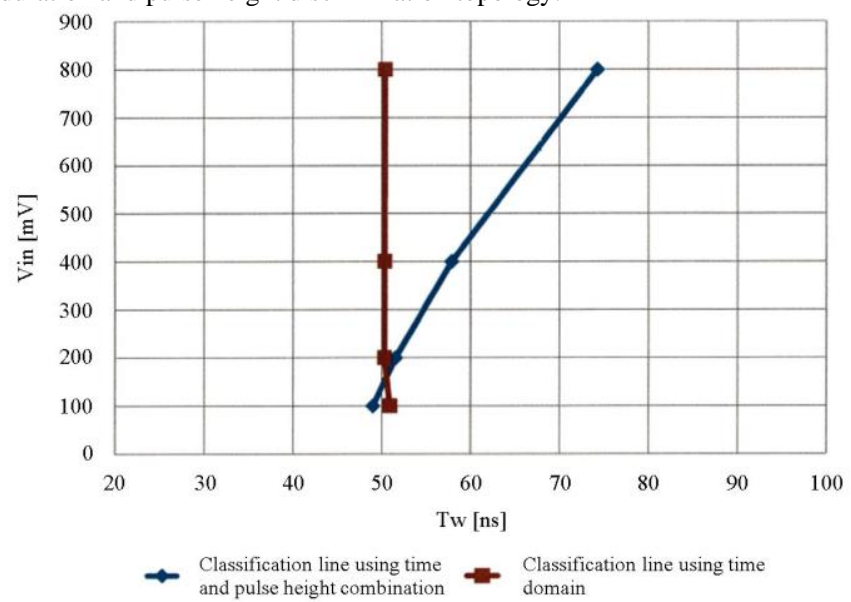

Fig. 10. Measured results of the classification curve in the height - time span, red curve using time duration topology, blue curve using the combined heighttime topology.

\section{CONCLUSIONS}

An analog topology that can discriminate between fast and slow pulses from plastic scintillators was developed. Using the height of the pulse as a restraining factor and combining it with a time domain PSD, the topology provides enhanced discrimination capabilities in inadequate light collection structures. The topology is not based on high frequency samplers or complex algorithms; hence, it has advantages both in power consumption and in processing time. The absence of long processing time ensures shorter dead time. By adjusting a few parameters in the electronics, the topology can easily be adapted for different detectors with different time constants and light yield coefficients.

\section{REFERENCES}

[1] D. H. Wilkinson, "The Phoswich-A Multiple Phosphor", Review of Scientific Instruments, vol. 23, no. 8, Aug. 1952, 10.1063/1.1746324.

[2] Matthew J.I.Balmer, " Comparative analysis of pulse shape discrimination methods in a Li-6 loaded plastic scintillator ", Nuclear Instruments and Methods in Physics Research Section A, Vol. 788, Jul 2015, 10.1016/j.nima.2015.03.089.

[3] C.S. Sosa, " Comparison of analog and digital pulse-shapediscrimination systems", Nuclear Instruments and Methods in Physics Research Section A, Vol. 826, May 2016, 10.1016/j.nima.2016.03.088.

[4] T. S. Sanderson, " Machine learning for digital pulse shape discrimination", 2012 IEEE Nuclear Science Symposium and Medical Imaging Conference Record (NSS/MIC), Oct. 2012, 10.1109/NSSMIC.2012.6551092.

[5] P. Holl, "Deep learning based pulse shape discrimination for germanium detectors", The European Physical Journal C, Vol. 79. no. 450, Jun. 2019, 10.1140/epjc/s10052-019-6869-2.

[6] M.Flaska, " Identification of shielded neutron sources with the liquid scintillator BC-501A using a digital pulse shape discrimination method",
Nuclear Instruments and Methods in Physics Research Section A, Vol. 577, Jul 2007, 10.1016/j.nima.2007.04.141.

[7] M.Nakhostin, " Application of digital zero-crossing technique for neutron-gamma discrimination in liquid organic scintillation detectors", Nuclear Instruments and Methods in Physics Research Section A, Vol. 621, Sep. 2010, 10.1016/j.nima.2010.06.252. 